\title{
Erratum: Shrinking of Rapidly Evaporating Water Microdroplets \\ Reveals their Extreme Supercooling [Phys. Rev. Lett. 120, 015501 (2018)]
}

Claudia Goy, Marco A. C. Potenza, Sebastian Dedera, Marilena Tomut, Emmanuel Guillerm, Anton Kalinin, Kay-Obbe Voss, Alexander Schottelius, Nikolaos Petridis, Alexey Prosvetov, Guzmán Tejeda, José M. Fernández, Christina Trautmann, Frédéric Caupin, Ulrich Glasmacher, and Robert E. Grisenti

(Received 22 February 2018; published 23 March 2018)

DOI: $10.1103 /$ PhysRevLett.120.129901

Figure 1 of the published Letter displays a misleading scale bar because the stroboscopic image of the stream of water droplets was actually not calibrated. This scale bar had the sole purpose of indicating the jet dimensions in an approximate way. A calibrated image with the correct scale bar is shown in Fig. 1 below. The results and conclusions of the Letter are not affected by the correction.

We are indebted to H. Pathak, A. Späh, L. G. M. Pettersson, and A. Nilsson for pointing to discrepancies that arose from the inaccurate scale bar.

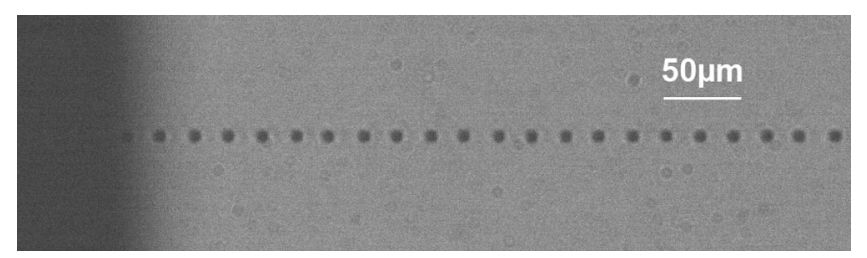

FIG. 1. Calibrated stroboscopic image of the periodic train of uniform water droplets generated during the Raman scattering experiments described in the original Letter. 